\title{
Salvatorische Klauseln in AGB
}

Arnold F. Rusch *

Das gesamte AGB-Korrektiv steht auf dem wichtigen Pfeiler des Verbots der geltungserhaltenden Reduktion. Dieses Verbot lässt sich nicht dadurch umgehen, dass man in den AGB nichtige Klauseln mit Ersatzklauseln zu heilen versucht. Die salvatorische Klausel hat deshalb in AGB nichts verloren.

Le contrôle des conditions générales exige la nullité des clauses abusives sans adaptation du contenu : l'application du principe de la réduction du contenu pour permettre son maintien est exclue. L'utilisateur de conditions générales ne peut point contourner ce principe par la voie des clauses salvatrices.

I. Was ist eine salvatorische Klausel? 74

II. Weshalb ist die salvatorische Klausel in AGB besonders schädlich? 74

III. Welche Typen salvatorischer Klauseln existieren und wie sieht das

Korrektiv dazu aus? ............................................................................. 76

1. Einfache salvatorische Klauseln .....................................................76

2. Abstrakte Freizeichnungen ........................................................... 76

3. Aufgespaltete Freizeichnungen .................................................... 78

4. Klauselhierarchien ................................................................. 79

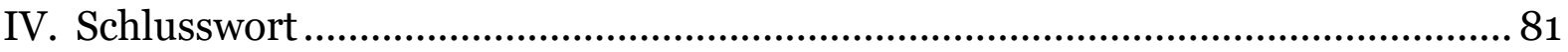

Zitiervorschlag: Arnold F. Rusch, Salvatorische Klauseln in AGB, in: sui-generis 2016, S. 73

URL: $\quad$ sui-generis.ch/25

DOI: $\quad$ https://doi.org/10.21257/sg.25

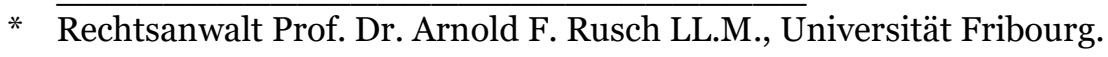

Dieses Werk ist lizenziert unter einer Creative Commons Namensnennung - Weitergabe unter gleichen Bedingungen 4.0 International Lizenz. 


\section{Was ist eine salvatorische Klausel?}

1 Die salvatorische Klausel will eine nichtige Klausel heilen und retten, indem sie einen Ersatz dafür vorsieht. Sie existiert in vielen Formen. Die schwächste Form ist die Neuverhandlungspflicht für den Fall, dass eine Klausel nichtig sein sollte. Intensivere Formen der salvatorischen Klausel sehen für den Fall der Nichtigkeit einzelner Klauseln fertig formulierte Ersatzlösungen vor. Diese eher plumpen Varianten stehen neben verdeckten Formen der salvatorischen Klausel, die beispielsweise eine Freizeichnung im gesetzlich maximal zulässigen Umfang vorsehen. Eine ähnliche Wirkung erzielen AGBRegelwerke, die eine Hierarchie der Normen im Falle eines Widerspruchs festlegen.

\section{Weshalb ist die salvatorische Klau- sel in AGB besonders schädlich?}

2 Es ist ein ganzer Strauss von Argumenten, die gegen die salvatorischen Klauseln sprechen:

Erstens liegt darin ein Verstoss gegen das Verbot der geltungserhaltenden Reduktion in AGB. Die herrschende Lehre bejaht das Verbot der geltungserhaltenden Reduktion im AGB-Bereich. ${ }^{1}$ Dieses Verbot

\footnotetext{
Peter Gauch/Walter Schluep/Jörg Schmid/Susan Emmenegger, Schweizerisches Obligationenrecht Allgemeiner Teil, Band I, 10. A., Zürich 2014, N 1155b; CHK-Lorenza Ferrari Hofer/David Vasella, in: Marc Amstutz et al. (Hrsg.), Handkommentar zum Schweizer Privatrecht, 3. A., Zürich 2016, UWG 8 N 14; BSK UWG-Florent Thouvenin, in: Reto M. Hilty/Reto Arpagaus (Hrsg.), Basler Kommentar Bundesgesetz gegen den unlauteren Wettbewerb, Basel 2013, UWG 8 N 146, m.H. auf abweichende Meinungen; Arnold F. Rusch, Schadensabwälzungsklauseln in der Inhaltskontrolle, SZW 2012, 439 ff., 443; Thomas Koller, Art. 8 UWG: Eine Auslegeordnung, in: Susan Emmenegger (Hrsg.), Das Bankkonto, Po-
}

geht auch aus dem Urteil des Bundesgerichts 4A_404/2008 vom 18. Dezember 2008, E. 5.6.3.2.1 klar hervor. Das Bundesgericht hat zuvor in E. 5.6.3.1 festgehalten, dass die geltungserhaltende Reduktion die Regel bildet - für Individualvereinbarungen, was ohne Zweifel richtig ist. In E. 5.6.3.2.1 hat es für die Verletzung zwingender Normen in allgemeinen Geschäftsbedingungen aber eine klare Ausnahme gemacht: «Denn die in der Lehre vertretene Ablehnung einer geltungserhaltenden Reduktion überzeugt jedenfalls schon insoweit, als der Nichtigkeitsgrund in einem Verstoss gegen eine zwingende Norm zum Schutz der schwächeren Vertragspartei liegt und die mangelhafte Klausel in vorgedruckten $A G B$ enthalten ist, in denen in einer Weise erheblich von der gesetzlichen Ordnung abgewichen wird, dass die Vermutung nahe liegt, es werde damit gezielt der Schutzzweck derselben unterlaufen (...).» Das Bundesgericht hat damit die unmittelbar vor dieser Erwägung erwähnte Voraussetzung verworfen, wonach für die Ganznichtigkeit eine Verletzung des (alten) Art. 8 UWG vorliegen müsse. Liegt eine Verletzung des alten oder des neuen Art. 8 UWG vor, dann muss die bundesgerichtliche Aussage folgerichtig a fortiori gelten. All diese Gedanken wären vollkommen wertlos, wenn man die geltungserhaltende Reduktion über die Hintertür der salvatorischen Klausel wieder in die AGB schmuggeln kann. Nach allen Regeln der Logik kann es nicht angehen, dass man mit vorformulierten Klauseln den Schutz vor vorformulierten Klauseln umgehen kann - sie müssen sich ihrerseits dem AGB-Korrektiv unterziehen. Der schädli-

licy - Inhaltskontrolle - Erwachsenenschutz, Basel 2013, 17 ff., 66 . 
che Effekt der salvatorischen Klausel liegt insbesondere darin, dass die AGBVerwender einen Anreiz erhalten, übermässige Klauseln aufzunehmen - im Wissen, dass sie im Nichtigkeitsfalle dennoch den maximal zulässigen Inhalt für sich sichern können.

kommt zweitens: Mit den salvatorischen Klauseln und der damit verbundenen geltungserhaltenden Reduktion bleiben alle missbräuchlichen Klauseln weiterhin im Umlauf. Eine funktionierende AGB-Kontrolle muss jedoch wollen, dass die missbräuchlichen Klauseln auch tatsächlich verschwinden - ansonsten sie gegenüber nichtsahnenden Konsumenten, die sich dadurch beeindrucken lassen, weiterhin Verwendung finden. Die europäische Rechtsprechung hat dies längst begriffen und jegliche geltungserhaltende Reduktion in AGB untersagt. ${ }^{2}$ Wenn man die Änderung des Art. 8 UWG als autonomen Nachvollzug des Schweizer Rechts erachtet - und dafür gibt es Hinweise - gehört diese Rechtsprechung gleich zum Gesetz dazu. 3

Drittens lassen sich AGB kaum noch erfassen, wenn man deren Umfang als Leser selber beurteilen muss. Dafür existieren mehrere Beispiele: Klauseln, die eine Befreiung oder Auferlegung von Pflichten «soweit gesetzlich zulässig» vorsehen, auf einen Gesetzesartikel verweisen oder eine Ersatzlösung für den Nichtigkeitsfall

2 EuGH, Urteil vom 14.6.2012 - C-618/10 (Banco Español de Crédito SA/Joaquín Calderón Camino), NJW 2012, 2257 ff., 2260, N 69-71; EuGH, Urteil vom 30.5.2013 - C-488/11 (Dirk Frederik Asbeek Brusse u. a./Jahani BV), NJW 2013, 2579 ff., 2582, N 57-60 und EuGH, Urteil vom 30.4.2014 - C-26/13 (Kásler ua/OTP Jelzálogbank Zrt), NJW 2014, 2335 ff., 2339, N 79.

3 Vgl. dazu die Hinweise ob und in FN 18. vorsehen, sind für Konsumenten in ihrer Tragweite nicht fassbar. Die Verständlichkeit der AGB bildet das materielle $\mathrm{Zu}-$ gänglichkeitserfordernis. Der Einbezug $\operatorname{der} A G B$ kann somit nicht nur an der fehlenden physischen Aushändigung oder am fehlenden Hinweis auf die AGB scheitern, sondern genauso an der fehlenden Verständlichkeit der AGB selbst. Die Gedanken zu den nachfolgenden Beispielen gehen auf dieses Transparenzgebot vertieft ein, das als Teil der Geltungskontrolle noch vor der Inhaltskontrolle gemäss Art. 8 UWG zur Anwendung gelangt. 4

6 Viertens verschiebt der AGB-Verwender mit der salvatorischen Klausel das Risiko des gesamten AGB-Korrektivs auf den Vertragspartner. Bei Nichtigkeit einer Klausel gilt das dispositive Recht, nicht eine Ersatzklausel.5 Die Risiken und Folgen des AGB-Korrektivs muss der AGBVerwender vollumfänglich tragen. Sie bilden das Gegenstück zum Vorteil der einseitigen Formulierungsfreiheit ${ }^{6}$ - ein Vorgehen gegen missbräuchliche Klauseln muss sich schliesslich auch lohnen. Die salvatorische Klausel führt deshalb immer auch zu einem erheblichen Missverhältnis zwischen den vertraglichen Rechten und Pflichten im Sinne des Art. 8 UWG.7

4 Vgl. Roman Perrig, in: Ernst A. Kramer/Thomas Probst/Roman Perrig, Schweizerisches Recht der Allgemeinen Geschäftsbedingungen, Bern 2016 (zitiert: Kramer/Probst/Perrig-Verfasser), N 141.

5 Vgl. Gauch/Schluep/Schmid/Emmenegger (FN 1), N 1155b; vgl. OLG Jena, Urteil vom 7.5.2014 2 U 114/14, BeckRS 2015, 05352, E. 3b, unter Hinweis auf BGH, Versäumnisurteil vom 22.11.2001 - VII ZR 208/o0, NJW 2002, 894 ff., 895 .

6 Siehe zu diesem Gedanken die Hinweise in FN 11.

7 Vgl. BGH, Urteil vom 8.5.2007 - KZR 14/04, NJW 2007, 3568 ff., 3570, N 24. 
7 Die nachfolgenden Gedanken widmen sich dem spezifischen Korrektiv zu den verschiedenen Typen der salvatorischen Klauseln in vier Eskalationsstufen.

\section{Welche Typen salvatorischer Klau- seln existieren und wie sieht das Korrektiv dazu aus?}

\section{Einfache salvatorische Klauseln}

8

«Sollten eine oder mehrere Bestimmungen dieser AGB unwirksam sein oder werden, so berührt oder beeinträchtigt dies die Gültigkeit und Vollstreckbarkeit der übrigen Bestimmungen nicht. Die Parteien verpflichten sich für diesen Fall, die unwirksame Regelung durch eine rechtswirksame Ersatzregelung zu ersetzen, die den wirtschaftlichen Absichten der ursprünglichen Regelung möglichst nahe kommt.»8 Diese Klausel bejaht eigentlich das Verbot der geltungserhaltenden Reduktion - im ersten Satz. Im zweiten Satz nimmt sie dieses wieder zurück und sieht einen Vorvertrag oder eine Pflicht zur Neuverhandlung vor. ${ }^{9}$ Betrachtet man die Situation realistisch und geht von der Nichtigkeit der in Frage stehenden Klausel zu Lasten des AGB-Verwenders aus, bedeutet dies, dass die Klausel im gerade noch zulässigen Umfang weitergelten soll. So haben in Deutschland mehrere Autoren diese Klausel gar nicht erst im Sinne einer Neuverhandlungspflicht verstanden

8 Ziff. 11 der AGB der Guave Studios GmbH (20.8.2016); vgl. die AGB von ricardo (20.8.2016), die eine einseitige Ergänzung vorsehen (Ziff. 13).

9 Vgl. dazu die weiteren Beispiele bei Lutz Michalski, Funktion, Arten und Rechtswirkungen von Ersetzungsklauseln, NZG 1998, 7 ff., 9; vgl. Jürgen F. Baur, Salvatorische Klauseln, in: Jürgen F. Baur/Rainer Jacobs/Manfred Lieb/PeterChristian Müller-Graff (Hrsg.), Festschrift für Ralf Vieregge, Berlin 1995, 31 ff., 35.
- was wohlgemerkt auch nicht zulässig wäre - sondern direkt als einseitiges Leistungsbestimmungsrecht ${ }^{10}$ des AGBVerwenders. Das spricht Bände und zeigt insbesondere, dass trotz unterschiedlicher Formulierung derselbe Inhalt wie bei der klassischen salvatorischen Klausel gilt, die eine Ersatzklausel direkt benennt. ${ }^{11}$ Dieser letztere Typus der salvatorischen Klausel kommt allerdings sehr selten vor. ${ }^{12}$ Für beide Klauseltypen gilt, dass man mit vorformulierten Klauseln den Schutz vor vorformulierten Klauseln nicht umgehen kann: Im AGB-Bereich gilt das «klauselfeste» Verbot der geltungserhaltenden Reduktion.

\section{Abstrakte Freizeichnungen}

9 Recht häufig versuchen AGB-Verwender, das Verbot der geltungserhaltenden Reduktion zu umgehen, indem sie eine vertragliche Einschränkung abstrakt maximal formulieren: "Die vertragliche oder ausservertragliche Haftung von Hilti im Zusammenhang mit Schäden, die auf Handlungen oder Unterlassungen der gesetzlichen Vertreter, Angestellten und Hilfspersonen von Hilti zurückzuführen sind, sowie

10 Vgl. die Hinweise bei Lutz Michalski/Volker Römermann, Die Wirksamkeit der salvatorischen Klausel, NJW 1994, 886 ff., 887; vgl. die Terminologie bei Wolfgang Jakob Hau, Vertragsanpassung und Anpassungsvertrag, Habil. Trier $2002=$ Jus Privatum, Band 83, Tübingen 2003, 401 f. das «Gestaltungsrechtsmodell», bei dem der AGB-Verwender die Ersatzklausel einseitig definiert, steht dem «Anspruchsmodell» gegenüber, bei dem beide Parteien bei der Suche nach einer Ersatzklausel mitwirken müssen. Richtigerweise behandelt Hau beide Modelle gleich.

11 Vgl. MK-Jürgen Basedow, Münchener Kommentar, 7. A., München 2016, BGB 306 N 30; vgl. BGH, Versäumnisurteil vom 22.11.2001 - VII ZR 208/o0, NJW 2002, 894 ff., 895; vgl. OLG München, Urteil vom 19.6.2008 - U (K) 4252/o7, BeckRS 2008, 12473, E. 1.a.cc.

12 Vgl. die Formulierungsbeispiele bei Michalski (FN 9), NZG 1998, 8. 
für die persönliche vertragliche oder ausservertragliche Haftung dieser Personen wird ausgeschlossen, soweit dies gesetzlich zulässig ist.»13 Auf den ersten Blick wäre also alles in Ordnung - schliesslich verweisen die AGB gerade auf das gesetzlich noch zulässige Mass. Dazu schreibt Perrig: «Einerseits ist dem Kunden nicht zuzumuten, die massgeblichen gesetzlichen Vorschriften zu ermitteln; anderseits kann er die einschlägigen Rechtsnormen - selbst wenn er sie auffinden würde - aufgrund ihres rechtstechnischen Charakters nicht in ihrer praktischen Tragweite einschätzen. Der Kunde wird durch solche Klauseln im Unklaren gelassen, welche Rechte ihm zustehen und welche Pflichten ihm aufgebürdet werden.»14 Angesichts der gerichtlich noch nicht geklärten Tragweite des neuen Art. 8 UWG könnte man dem Argument auf den Leim kriechen, dass die salvatorische Klausel gerade deswegen für die AGB-Verwender notwendig sei. Dieses Argument ist jedoch falsch, weil der $A G B$-Verwender die Verantwortung für einen verständlichen und gültigen AGB-Text trägt. 15 Dieses Risiko gehört zur einseitigen Gestaltungsmacht der AGB dazu. Auch die deutsche Lehre betrachtet diese Variante der salvatorischen Klausel als nichtig: Die Klausel verstosse

$13 \overline{\text { Ziff. } 13 \text { der AGB von Hilti (Schweiz) AG und }}$ Ziff. 12 der AGB für Dienstleistungen der Swisscom (beide 20.8.2016).

14 Roman Perrig, Die AGB-Zugänglichkeitsregel, Diss. Basel $2011=$ Basler Studien zur Rechtswissenschaft, Band 102, 240 (zit. Perrig); vgl. Kramer/Probst/Perrig-Perrig (FN 4), N 147; beide Zitate Perrigs zeigen, dass auch der Verweis auf Gesetzesnormen intransparent sind (vgl. dazu MK-Basedow [FN 11], BGB 305 N 74 f.).

15 Perrig (FN 14), 241; MK-Basedow (FN 11), BGB 305 N 75; vgl. Hubert Schmidt, in: Heinz Georg Bamberger/Herbert Roth (Hrsg.), Beck'scher Online-Kommentar, 39. A., München 2016, BGB $306 \mathrm{~N}$ 19; a.A. Hau (FN 10), $402 \mathrm{f}$. gegen das Transparenzgebot. ${ }^{16}$ Das deutsche Recht erwähnt dieses in $\S 307$ Abs. 1 BGB: «Bestimmungen in Allgemeinen Geschäftsbedingungen sind unwirksam, wenn sie den Vertragspartner des Verwenders entgegen den Geboten von Treu und Glauben unangemessen benachteiligen. Eine unangemessene Benachteiligung kann sich auch daraus ergeben, dass die Bestimmung nicht klar und verständlich ist.»

10 Existiert dieses Verständlichkeitsgebot auch in der Schweiz? Satz 1 der Bestimmung gleicht dem neuen Art. 8 UWG aufs Haar. Satz 2 sucht man im UWG und im OR indes vergeblich. Mit der Hinzufügung von Satz 2 im Rahmen der Schuldrechtsmodernisierung wollte der deutsche Gesetzgeber aber nicht etwa die Rechtslage verändern, sondern lediglich die Rechtsprechung nachführen, die auch in Umsetzung der EU-Richtlinie erging. ${ }^{17}$ Diese hält in Art. 5 fest, dass alle Klauseln «stets klar und verständlich abgefasst» sein müssen. Nun gibt es viele Stimmen, die in Art. 8 UWG einen autonomen Nachvollzug der EU-Richtlinie sehen. Der praktisch identische Wortlaut legt dies tatsächlich nahe. ${ }^{18}$ Dann aber gilt die gesamte Richtlinie mitsamt Rechtsprechung als mitübernommen, weil der Nachvollzug sonst auf halber Strecke ste-

$16 \overline{\text { MK-Basedow (FN 11), BGB } 306 \mathrm{~N} \text { 30, m.w.H. }}$ und $\S 305 \mathrm{~N} 75$; vgl. auch BGH, Beschluss vom 5.3.2013 - VIII ZR 137/12, NJW 2013, 1668 f., 1668.

17 BT-Drs. 14/6040 vom 14.5.2001, 153 f.; zur Geschichte des Transparenzgebots siehe auch Kent D. Lerch, Verständlichkeit als Pflicht? In: Kent D. Lerch (Hrsg.), Die Sprache des Rechts, Band I, Recht verstehen, Berlin 2004, 239 ff., $240 \mathrm{ff}$.

18 Vgl. dazu Kramer/Probst/Perrig-Probst (FN 4), N 448, m.w.H. und Hubert Stöckli, Der neue Art. 8 UWG - offene Inhaltskontrolle, aber nicht für alle, BR 2011, 184 ff., 186. 
henbleibt. ${ }^{19}$ Unabhängig von diesem Argument lässt sich das Transparenzgebot aber auch direkt in das Erfordernis der Übernahme der AGB und in den Art. 8 $U W G$ hineininterpretieren. Der Einbezug unverständlicher oder schwer verständlicher AGB scheitert nicht etwa nur im Rahmen der Unklarheitenregel, sondern schon am Erfordernis der zumutbaren Kenntnisnahme. ${ }^{20}$ Was nicht klar und deutlich daherkommt, ist bei der Übernahme ähnlich schwierig zugänglich wie unleserliche, nicht ausgehändigte oder auf einem anderen Kanal ausgehändigte AGB. Liegt dann auch eine Verletzung des Art. $8 U W G$ vor? Wer die Zugänglichkeitsregel verletzt, verletzt erst recht den neuen Art. 8 UWG: Erstens lässt sich gut begründen, dass auch unverständliche Regeln ein ungerechtfertigtes Missverhältnis zwischen vertraglichen Rechten und Pflichten schaffen. Dies, weil man Klauseln in den Vertrag nimmt, die man nicht versteht und damit auch nicht will. In eine unverständliche Klausel lässt sich alles Mögliche hineininterpretieren, weshalb sie bei der Inhaltskontrolle ebenfalls scheitern muss. ${ }^{21}$ Hinzu kommt bei den

19 Zu diesem Prinzip BGE 137 III 226 ff., 229, m.w.H.

20 Perrig (FN 14), 217 ff., insb. 226 ff.; KG, Urteil vom 11.11.1998 - Kart U 387-98, NJW-RR 1999, 1659 ff., 1660 f.; vgl. OLG Schleswig, Rechtsentscheid vom 27.3.1995 - 4 RE-Miet 1/93, NJW 1995, 2858 ff., 2859; vgl. zu den Grundlagen der Verständlichkeit Hans-Peter Schwintowski, Verständlichkeit von Allgemeinen Geschäftsbedingungen - eine empirische Studie, HAVE 2006, $218 \mathrm{ff}$., $221 \mathrm{ff}$. und Lerch (FN 17), $244 \mathrm{ff}$.

21 Vgl. ansatzweise Kramer/Probst/Perrig-Perrig (FN 4), N 141 Fn. 448; vgl. die Erfassung schwer verständlicher Klauseln als missbräuchliche Klauseln bei Thomas Pfeiffer, in: Wolf/Lindacher/Pfeiffer, AGB-Recht, 6. A., München 2013 (zit. Wolf/Lindacher/Pfeiffer-Verfasser), 7. Teil, Richtlinie 93/13/EWG, Art. 5 N 2224, unter Hinweis auf EuGH (Erste Kammer), Urteil vom 26.4.2012 - C-472/10 (Nemzeti Fog- salvatorischen Klauseln, dass sich das gesamte Risiko des AGB-Korrektivs vom Verwender auf den Konsumenten verschiebt und die Ersatzanwendung des dispositiven Rechts ausbleibt. ${ }^{22}$ Der Grund liegt aber zweitens auch in der Folgenerwägung, dass sich solche Verstösse sonst nicht via Verbandsklageverfahren nach Art. 10 UWG durchsetzen lassen, da sich dieses möglicherweise auf Verletzungen des Art. 8 UWG beschränkt. ${ }^{23}$

\section{Aufgespaltete Freizeichnungen}

11 Ist es möglich, das Verbot der geltungserhaltenden Reduktion zu überlisten, indem man den maximalen Klauselinhalt in sämtliche Teilinhalte aufspaltet? So wäre es beispielsweise denkbar, sich zuerst von der Haftung für Vorsatz freizuzeichnen. Die zweite Klausel enthielte die Freizeichnung für grobes Verschulden, die dritte für mittleres. Die letzte Klausel würde die Haftung für leichtes Verschulden wegbedingen. Die ersten beiden Klauseln wären nichtig (Art. 100 Abs. 1 OR). Der AGB-Verwender könnte nichtsahnenden Kunden alle vier Klauseln entgegenhalten, solange sie sich davon beeindrucken lassen. Wehren sie sich, fallen die nichtigen Klauseln - aber nur diese - ausser Betracht. Da jede Klausel für sich existiert, könnte der AGBVerwender sich nach wie vor auf die dritte und die vierte Klausel stützen, die Art. 100 OR und dem AGB-Korrektiv viel-

yasztóvédelmi Hatóság/Invitel Távközlési Zrt), EuZW 2012, 786 ff., 787, N 27-31.

22 Vgl. die Hinweise bei FN 5 und 11.

23 Wolf/Lindacher/Pfeiffer-Pfeiffer (FN 21), Art. 5 $\mathrm{N} 24$ lässt zu Recht die Anrufung des Transparenzgebots auch im Verbandsklageverfahren zu; ebenso Arnold F. Rusch/Andreas Schirrmacher, Konsumentenorganisationen im AGB-Streit, ZBJV 2013, 683 ff., 688. 
leicht standhalten. Die deutsche Rechtsprechung hat den sog. blue pencil test übernommen, der in diesem Fall auch keine Remedur leistet: "Die inhaltliche Trennbarkeit einer Klausel und damit ihre Zerlegung in einen inhaltlich zulässigen und einen inhaltlich unzulässigen Teil ist immer dann gegeben, wenn der unwirksame Teil der Klausel gestrichen werden kann, ohne dass der Sinn des anderen Teils darunter leidet (sog. blue-pencil-test); $o b$ beide Bestimmungen den gleichen Regelungsgegenstand betreffen ist dabei unerheblich (...).» ${ }^{24}$ Dieser Test generiert jedoch nicht nur im vorgenannten Beispiel untaugliche und zufällige Ergebnisse. Er bewirkt im Ergebnis gerade eine geltungserhaltende Reduktion, weil er auch einzelne Satzteile oder gar einzelne Wörter als streichbar und damit als Klauseln betrachtet. Ob der AGB-Verwender die Haftung generell wegbedingt, die einzelnen Verschuldensgrade gesondert und damit streichbar aufzählt oder die Klausel in mehrere Klauseln aufspaltet, kann im Ergebnis keine Rolle spielen. Die Zufälligkeit der dadurch generierten Resultate darf nicht zu Lasten der Konsumenten gehen.25 Bereits gibt es Stimmen, die dieses Vorgehen beim Verfassen der AGB empfehlen, ${ }^{26}$ was alle Alarmglocken läuten lässt. Notfalls könnte auch das Verbot des Rechtsmissbrauchs dem Unfug des Aufspaltens einzelner Klauseln ein Ende

24 BGH, Urteil vom 10.10.2013 - III ZR 325/12, NJW 2014, $141 \mathrm{ff} ., 142$, N 14.

25 Andreas Geroldinger, Klauselbegriff und «blue pencil test» in der AGB-Rechtsprechung, ALJ 2/2015, 196 ff., 207 ff.; Hanns Fitz, Zur «geltungserhaltenden Reduktion» überschiessender AGB-Klauseln, in: Oswin Martinek/Gustav Wachter (Hrsg.), Arbeitsleben und Rechtsordnung, Festschrift Gerhard Schnorr, Wien 1988, 645 ff., 652.

26 Staudinger-Peter Schlosser, Berlin 2013, BGB $306 \mathrm{~N} 20$. setzen: «Wo der Missbrauch und die Umgehungsabsicht mit Händen zu greifen ist, sollte daher durch die Nichtigkeit des gesamten Klauselkomplexes Remedur geschaffen werden. Man braucht hierfür kein positiviertes Umgehungsverbot (...), sondern lediglich die Überlegung, dass derjenige, der offenbar auf die Akzeptanz einer weiterreichenden Klausel hofft, um sich andernfalls auf die geringere zurückziehen zu können, schuldhaft Intransparenz schafft und damit vorvertragliche Verhaltenspflichten verletzt. Er darf daher nicht besser gestellt werden als ein Verwender, der weniger fintenreich gegen das Gesetz verstösst.»27

\section{Klauselhierarchien}

12 Klauselhierarchien für den Fall widersprüchlicher Klauseln wirken gleich wie klassische salvatorische Klauseln, weisen aber eine darüber hinausreichende Problematik auf. Als Beispiel kann die SIA 118 dienen. Art. 21 SIA 118 regelt, welche Vertragsbestandteile im Falle eines Widerspruchs zueinander den Vorrang erhalten. Dadurch ist es erstens möglich, dass in untergeordneten Dokumenten enthaltene Individualabreden ihren Vorrang verlieren. ${ }^{28}$ Zweitens ergibt sich dadurch ein Widerspruch zu Art. 2 Abs. 2 SIA 118, der den Vorrang aller Individualvereinbarungen festhält. 29 Bei genauer Betrachtung liegt drittens eine der salvatorischen Klausel ähnliche Regel vor:30 Bei

27 Fitz (FN 25), 652.

28 Vgl. Peter Gauch, Der Werkvertrag, 5. A., Zürich 2011, N 288, 314, 316.

29 Vgl. Gauch (FN 28), N 288 und Urteil des Bundesgerichts 4C.420/2006 vom 3. August 2007, E. 4 .

30 Vgl. zu den Rangordnungsklauseln in der Vertragsurkunde Perrig (FN 14), 239 f. und konkreter zu Rangordnungen in den $A G B$ Stefan De- 
widersprüchlichen AGB ergibt sich der Vorrang aufgrund der Unklarheitenregel nämlich für die dem Verfasser weniger günstigere Regel, nicht für die gemäss AGB prioritär geltende Regel. ${ }^{31}$ Genauso ergibt sich der Vorrang für die individuell vereinbarte Klausel, nicht für die gemäss AGB vorrangige Klausel. Diese Probleme gelten für alle mündlichen Abreden, für alle nicht der Formvorschrift gemäss Art. 21 Abs. 3 SIA 118 entsprechenden Abreden und für alle Individualabreden, die in nachrangigen Dokumenten enthalten sind. Der Vorrang der Individualabrede und die Unklarheitenregel gelten absolut. Sie sind nicht Teil der Inhaltskontrolle, sondern Teil der Ermittlung des Vertragsinhalts. Derogiert eine vorformulierte Klausel diesen Grundsatz generell, steht sie im Widerspruch zur zwingenden gesetzlichen Ordnung, ${ }^{2}$ was die entsprechende Klausel nichtig macht und überdies a fortiori einen Verstoss gegen Art. 8 UWG darstellt.33 Nun könnte man einwenden, dank der Prioritätenregel ergebe sich gar keine Unklarheit. Wiederum kann es aber nicht angehen, dass vorformulierte Klauseln spezifische Schutzinstrumente gegen vorformulierte Klauseln aushebeln - dafür bedürfte es schon eines bewussten Entscheids, den

ckers, Unwirksame VOB/B-Klauseln im Verbrauchervertrag, NZBau 2008, 627 ff., 628; vgl. auch Harry Schmidt, in: Ulmer/Brandner/Hensen, AGB-Recht, 11. A., Köln 2011, BGB 306 N 40, m.w.H.

31 Vgl. die zutreffenden Überlegungen bei Deckers (FN 30), NZBau 2008, 628; teilweise a.M. Claus von Rintelen, in: Burkhard Messerschmidt/Wolfgang Voit (Hrsg.), Privates Baurecht, 2. A., München 2012, BGB 631 N 72, m.w.H. und BGH, Urteil vom 21. Juni 1990 - VII ZR 308/89, NJW 1990, 3197 ff., 3198, m.w.H.

32 Vgl. die identischen Ideen im deutschen Recht bei Wolf/Lindacher/Pfeiffer-Dammann (FN 21), BGB 310 Anh. S 98 ff.

33 Rusch/Schirrmacher (FN 23), ZBJV 2013, 688. die Parteien individualvertraglich fällen müssen. Die Prioritätenklausel hebt die Unklarheit der AGB ohnehin nur vordergründig auf - zum Preis einer unverständlichen und auslegungsbedürftigen Kaskade von Klauseln. Somit verstösst die Klauselhierarchie auch gegen das oben erwähnte Transparenzgebot. Mit Blick auf das enorme Missbrauchspotential und die gänzlich verlorene Transparenz der Klauselwerke34 ist deshalb ein Verstoss gegen Art. 8 UWG zu bejahen, weil sie zusätzlich das Verbot der geltungserhaltenden Reduktion, die Unklarheitenregel und den Vorrang der Individualvereinbarung aufheben. 35

13 Hinzu kommt: Für die Auslegung des Art. 8 UWG spielt die Übernahme des EU-Rechts eine entscheidende Rolle. Gerade Art. 1 lit. $\mathrm{n}$ des Anhangs zur Richtlinie 93/13/EWG erteilt Schriftformklauseln eine Absage, wenn «die Verpflichtung des Gewerbetreibenden zur Einhaltung der von seinen Vertretern eingegangenen Verpflichtungen eingeschränkt wird oder diese Verpflichtung von der Einhaltung einer besonderen Formvorschrift abhängig gemacht wird». Häufig dürften sich mündliche Individualabreden mit Vertretern des Bauunternehmers ergeben. Die Nichtgeltung und die Nichtigkeit der Klauseln sind damit gut begründbar.

$34 \overline{\text { Thomas Pfeiffer, Was kann ein Verbraucher? Zur }}$ Relevanz von Informationsverarbeitungskapazitäten im AGB-Recht und darüber hinaus, NJW 2011, 1 ff., 7, mit Hinweis auf BGH, Urteil vom 21.11.1985 - VII ZR 22/85, NJW 1986, 924 ff.

35 Vgl. die Gedanken bei Wolfgang Fell, Hintereinandergeschaltete Allgemeine Geschäftsbedingungen, ZIP 1987, 690 ff., 692. 


\section{Schlusswort}

14 Salvatorische Klausel sind sinnvoll - in Individualverträgen. In AGB weisen sie hingegen einen besonders schädlichen Effekt auf. Die obige Analyse der salvatorischen Klauseln in vier Eskalationsstufen - von der simplen Ersatzklausel über die aufgespaltete Freizeichnungsklausel zur vorformulierten Klauselhierarchie hat gezeigt, dass praktisch das gesamte AGB-Korrektiv zur Anwendung gelangt. Wer diese Klauseln in seine AGB aufnimmt, muss sich vor Gericht eines Besseren belehren lassen. Falls dies aufgrund der rationalen Prozess-Apathie in der Schweiz nicht stattfindet, lese ich ihm gerne persönlich die Leviten: eine salvatorische Belehrung also. Diese ist generell zulässig! 